\title{
Improving the management of acute pancreatitis in a district general hospital
}

\author{
Nicola Crowther, Mia Kahvo, Prem Chana \\ Great Western Hospital, Swindon, United Kingdom
}

\begin{abstract}
Acute pancreatitis is a common problem seen in the United Kingdom, with an incidence of 56.6 per 100,000 population.[1,2,3] Optimising management has been shown to reduce mortality and morbidity, and the British Society of Gastroenterology (BSG) published revised guidelines in 2005 to standardise treatment for this potentially life threatening condition.[4]
\end{abstract}

The aim of this quality improvement project was to investigate and improve the initial management of acute pancreatitis in patients presenting to the Great Western Hospital (GWH) in Swindon between November 2012 and July 2013.

Patients presenting to the surgical team during this time with a diagnosis of acute pancreatitis were identified for the initial data collection. Notes were prospectively reviewed and data collected allowing a comparison between management in GWH against BSG guidelines. Following this stage, a pro forma based on the 2005 guidelines was created and implemented, with the aim of raising awareness and standardising care among surgical staff. Following implementation of the pro forma, data collection was repeated between May and June 2013 to assess the impact of the intervention.

Results revealed an improvement from $93 \%$ to $100 \%$ of patients receiving the correct diagnosis within 24 hours of presentation. Severity stratification within 48 hours of diagnosis improved from $75 \%$ to $88 \%$ and identification of aetiology also improved from $64 \%$ to $74 \%$.

The implementation of an acute pancreatitis management protocol and education of junior surgical staff has been shown to improve compliance with BSG guidelines at the GWH, and ultimately aims to improves patient care and outcomes.

\section{Problem}

Awareness of the BSG guidelines was variable among surgical teams within the department of surgery at the Great Western Hospital (GWH). There was a significant variation in the management of acute pancreatitis with subsequent poor outcomes, particularly recurrent admissions and a long length of stay. This may be related to there being no dedicated upper gastrointestinal surgery trained surgeons in GWH. In addition, no trust-wide guidelines or protocols were present to aid staff in providing highquality, standardised care for the initial management of acute pancreatitis.

\section{Background}

Acute pancreatitis is a significant problem in the United Kingdom, with an incidence of 150 to 420 cases per million population, with a corresponding increase in its risk factors. $[1,2,3]$ It represents a significant proportion of workload for surgical departments across the country and accounts for approximately $3 \%$ of admissions to hospital with acute abdominal pain.[5] There is a wide spectrum of severity of the disease and cases are classified using a variety of severity scoring systems as mild, moderate, or severe, with severe disease carrying a significant mortality of up to $40 \%$ seen with infected pancreatic necrosis.[4] It has been shown that recognition of severity coupled with early implementation of organ specific support with endoscopic, radiological, and surgical interventions can help to reduce morbidity and mortality. $[1,4]$

The British Society of Gastroenterology (BSG) defined evidencebased national guidelines on optimal management of acute pancreatitis in 1998, with revised guidelines being subsequently published in 2005.[1,4] These were developed in response to multicentred audits highlighting deficiencies in the management of the disease, with a lack of standardised protocols both within and between institutions.[6] The BSG guidelines provide a framework for clinicians to follow and set audit goals.[4] Please see the supplementary file attached for guidelines (box 1).

This potentially life-threatening condition requires optimal management, and having a framework to follow in GWH will improve doctors' confidence and ability to provide high-quality, evidence based care. Other UK units have successfully carried out quality improvement projects to introduce care pathways resulting in improved compliance with the BSG guidelines.[7]

\section{Baseline measurement}

The baseline measurement was taken by looking prospectively at patients admitted under the general surgical team at the GWH between November 2012 and January 2013 with a diagnosis of 
acute pancreatitis. The diagnosis required two out of three of clinical, biochemical, and radiological evidence of acute pancreatitis. Electronic and paper notes were reviewed to collect data on diagnosis, severity stratification, aetiology, mortality, imaging, and treatment.

Patients presenting with chronic pancreatitis were excluded. Data were compared to the BSG guidelines to assess compliance. The guidelines used for comparison can be seen in the supplementary file attached (box 2).

A total of 29 patients were identified for the baseline measurement. One patient was excluded from the study as they presented with an acute flare up of chronic pancreatitis. The results can be seen below:

- Correct diagnosis of acute pancreatitis within 24 hours: BSG guideline 100\%; GWH 93\%

- Severity stratification within 48 hours of admission: BSG guideline 100\%; GWH 75\%

- Severe acute pancreatitis should be managed initially in HDU or ICU: BSG guidelines $100 \%$; GWH $14 \%$. (of note only $29 \%$ of severe cases were discussed with ITU)

- Ultrasound scan within 24 hours of admission: 100\%; GWH 50\%

- Aetiology determined: BSG guidelines 80\%; GWH 64\%

- Definitive management of gallstone pancreatitis during admission or within two weeks of discharge: BSG 100\%, GWH 21\%

- ERCP for severe gallstone pancreatitis within 72 hours: BSG $100 \%$; GWH 0\%

- Mortality: BGS < 10\%; GWH 4\% (BSG for severe <30\%; GWH for severe $14 \%$ ).

See supplementary file: ds4118.pdf - "Box 1 and Box 2"

\section{Design}

When considering the underlying cause for the problem it became apparent that multiple interventions were required, with reasons to support each. The first would be to create an acute pancreatitis pro forma based on the BSG guidelines. This would be readily available to junior medical staff and its use would be compulsory for all patients diagnosed with acute pancreatitis in GWH. Once completed, the pro forma would be included as part of the patient's notes to encourage compliance with guidelines as well as ensure accurate documentation.

The second stage was to raise awareness of the problem to the senior surgical staff at the GWH. The initial results would be presented to consultant surgeons at the monthly departmental meeting in order to gather senior support for the project and gain ideas for improvement of the pro forma design.
Awareness of the guidelines and the impact of deficiencies in management of patients with acute pancreatitis were noted to be variable among all medical staff when collecting the initial data and therefore it was apparent that education would be required. A teaching session on acute pancreatitis management, including the results from the baseline measurement, should be provided to the whole surgical team in GWH at a departmental meeting.

\section{Strategy}

PDSA cycle 1: The pro forma was initially presented to the surgical consultants within the trust alongside the initial results. Feedback was very positive and minor changes were made to the layout of the pro forma. An example of this was that in order to make the pro forma more user-friendly and easier to interpret it should be limited to one A4 page.

PDSA cycle 2: An education and awareness session was held for all the surgical teams in GWH with teaching on acute pancreatitis recognition and management along side the proposal for the implementation of the pro forma. Feedback was collected and was again positive.

PDSA cycle 3: The "acute pancreatitis pro forma" was made available in the surgical admissions unit and emergency department to make it easily accessible to junior staff providing primary assessment. This was trialled for one month to allow staff to familiarise themselves with the pro forma and feedback suggestions for improvement. The general consensus from medical staff was that the pro forma provided them with a prompt to carry out the appropriate initial investigations and management required at the time of diagnosis. Feedback from senior surgical staff was that it clearly displayed the important and relevant information required on ward rounds. One of the recurring issues was there was no space to re-score the predicted severity at 48 hours. In addition, a further suggestion was to make space for all the laboratory results on admission to be documented on the same sheet.

PDSA cycle 4: The issues identified in PDSA cycle 3 were taken on board and changes were made. The improved pro forma was distributed for surgical teams to use. Feedback was positive and no negative issues were raised. Data were prospectively collected for two months on all patients presenting to the trust with acute pancreatitis. This was again compared to BSG guidelines to assess compliance and demonstrated an improvement.

PDSA cycle 5: The pro forma was rolled out across the whole GWH and made available to all staff on the trust intranet.

Please see supplementary file for pro forma.

\section{Results}

After the aforementioned interventions, including education, awareness, and pro forma data to assess compliance in the trust against BSG guidelines were collected: 
- Correct diagnosis of acute pancreatitis within 48 hours of admission: BSG guideline - 100\%; round 1 - 93\%; round 2 - 100\% (p-value 0.11)

- Severity stratification within 48 hours of admission: BSG guideline - 100\%; round 1 - 75\%; round 2 - $88 \%$ (p-value 0.17 )

- Severe acute pancreatitis managed initially in an HDU/ICU setting BSG guideline - 100\%; round 1 - 14\%; round 2 - $8 \%$ ( $p$-value 0.65 )

- Severe acute pancreatitis case discussed with ICU: round 1 $29 \%$; round 2 - $92 \%$ (p-value 0.003 )

- Ultrasound within 24 hours: BSG guideline - 100\%; round 1 - 50\% round 2 - 53\% (p-value 0.81 )

- Aetiology determined: BSG guideline - $80 \%$; round 1 - $64 \%$; round 2 - 74\% (p-value 0.43 )

- Definitive management of gallstone pancreatitis during the same admission or within two weeks of discharge: BSG guideline - $100 \%$; round $1-21 \%$; round 2 - $0 \%$

- Urgent ERCP (within 72 hours) for severe gallstone pancreatitis, dilated bile ducts or cholangitis: BSG guideline - $100 \%$; round 1 $0 \%$; round 2 - $8 \%$

- Mortality in severe pancreatitis: BSG guideline - $<30 \%$; round 1 $14 \%$; round 2 - $0 \%$

- Overall mortality: BSG guideline - $<10 \%$; round 1 - 4\%; round 2 $3 \%$ (p-value 0.91).

See supplementary file: ds4119.pdf - "Acute Pancreatitis Pro Forma

\section{Lessons and limitations}

There were several lessons learnt during this project. Firstly, gaining the support and experience of senior staff allowed the project to develop more effectively and efficiently. The surgical consultants were able to support the education of junior staff and encourage the use of the pro forma while emphasising the importance of optimising initial patient management.

Secondly, smaller PDSA cycles are more effective than implementing one intervention as it allows adaptation of ideas to ensure the change is both beneficial to patient care and userfriendly. Engaging all grades in the surgical team through the evolution of the pro forma helped to gain accurate feedback and create an end product that is practical and easy to adopt while avoiding the burden of increasing workload.

One potential further improvement would be creating a formal questionnaire to gain feedback on the pro forma. Feedback was carried out by direct questions to selected staff in the third and fourth cycle. A formal questionnaire would have allowed us to gain objective feedback from all members of the surgical team.
There were two main limitations to this project. There was a relatively small sample size in both the baseline measurements and final results. Carrying out the project over a longer period would have allowed for large patient numbers and potentially more statistically significant results. The data compared compliance with the BSG guidelines and therefore it is not possible to comment on whether hospital stay and late complication rates were reduced. This would require a larger study that includes long-term patient outcome.

\section{Conclusion}

There has been a positive shift in compliance with BSG guidelines following this quality improvement project. In particular, it appears to have had a considerable effect on obtaining the diagnosis within 24 hours as well as documented severity scoring and aetiology.

Gallstone specific management has poorest compliance both at the beginning and end of this quality improvement project. This is likely to lead to increased morbidity for patients, increased readmission rates and the associated financial burden. This is likely to be related to there being no dedicated Upper Gl trained Surgeons in GWH. Further research is required to determine other influencing factors and consider interventions that aim to improve this.

The BSG guidelines state that all patients with severe pancreatitis should be managed in an HDU or ICU setting. Compliance with this guideline appears to be very poor with only $8 \%$ being admitted to ICU, but in practice this is not always possible due to bed availability and resources. However, the project did demonstrate a significant improvement in the number of patients with severe pancreatitis having a documented discussion with the ICU team (29\% to $92 \%$ ) although the decision was made that they did not require admission. This highlights that staff were better at recognising the potential severity of the condition and at documenting these discussions.

This quality improvement project appears to have made a positive impact on management of acute pancreatitis in a District General Hospital and has identified areas which require further work. There is always a need to refine and optimise systems and this project has shown that low-cost interventions can help to improve compliance with guidelines and ultimately improve patient care.

\section{References}

1. BSG Working Party. United Kingdom guidelines for the management of acute pancreatitis. Gut 1998;42 (suppl 2):S1-S13.

2. McKay CJ, Evans S, Sinclair M, Carter CR, Imrie CW. High early mortality rate from acute pancreatitis in Scotland, 1984-1995. Br J Surg 1999;86:1302-5.

3. Toh SK, Phillips S, Johnson CD. A prospective audit against national standards of the presentation and management of acute pancreatitis in the South of England. GUT 2000; 46:239-43.

4. UK Working Party on Acute Pancreatitis. UK guidelines for 
the management of acute pancreatitis. GUT 2005;54 (suppl 3):1-9.

5. De Dombal F. The acute abdomen: definitions, diseases and decisions. In: Diagnosis of Acute Abdominal Pain. 2nd edn. London: Churchill Livingston, 1991: 19-30.

6. Mann D, Hershman M, Hittinger R, et al. Multicentre audit of death from acute pancreatitis. Br J Surg 1994;81:890-3.

7. McCallum I, Hicks GJ, Attwood S, Seymour K. Impact of a care pathway in acute pancreatitis. Frontline Gastroenterol 2011; 2: 32-4.

\section{Declaration of interests}

Nothing to declare.

\section{Acknowledgements}

Nil 